\title{
Post-operative serum procalcitonin vs. C-reactive protein as a marker of post-operative infectious complications in pancreatic surgery: A systemic review and meta-analysis
}

\author{
Bhavin VASAVADA*, Hardik PATEL
}

Department of Hepatobiliary and Liver Transplant, Consultant Hepatobiliary and Liver Transplant, Ahmedabad, India

Introduction: The aim of this meta-analysis was to compare the diagnostic accuracy of C-reactive protein (CRP) and Procalcitonin (PCT) between postoperative days (POD) 3 to 5 in predicting infectious complications post pancreatic surgery.

Methods: A systemic literature search was performed using MEDLINE, EMBASE, and SCOPUS to identify studies evaluating the diagnostic accuracy of PCT and CRP as a predictor for detecting infectious complications between POD 3 to 5 following pancreatic surgery. A meta-analysis was performed using the random-effect model and pooled predictive parameters. Geometric means were calculated for PCT cut-offs.

Results: After applying inclusion and exclusion criteria 15 studies consisting of 2,212 patients were included in the final analysis according to Preferred Reporting Items for Systematic Reviews and Meta-Analyses guidelines. Pooled sensitivity, specificity, Area under the curve, and diagnostic odds ratio (DOR) for day 3 CRP were respectively $62 \%, 67 \% 0.772$, and 6.54 . Pooled sensitivity, specificity, Area under the curve, and DOR for day 3 PCT was respectively $74 \%, 79 \%, 0.8453$, and 11.03 . Sensitivity, specificity, Area under the curve and DOR for day 4 CRP were respectively $60 \%, 68 \%, 0.8022$, and 11.90 . Pooled sensitivity, specificity, and DOR of postoperative day 5 PCT level in predicting infectious complications were respectively $83 \%, 70 \%$, and 12.9. Pooled sensitivity, specificity, Area Under a ROC Curve, and diagnostic odds ratio were respectively 50\%, 70\%, 0.777, and 10.19 .

Conclusions: Post-operative PCT is better marker to predict post-operative infectious complications after pancreatic surgeries and post-operative day 3 PCT has highest diagnostic accuracy. 